Journal of Computations \& Modelling, Vol. 12, No. 1, 2022, 1-4

ISSN: $1792-7625$ (print), 1792-8850 (online)

https://doi.org/10.47260/jcomod/1211

Scientific Press International Limited

\title{
A Shortcut Model for the Suggested Approach in Mixed Zero-One Fuzzy Goal Programming
}

\author{
Maged G. Iskander ${ }^{1}$
}

\begin{abstract}
In this short paper, an equivalent model to the one that has been provided by Iskander [1] is presented. The required linearization constraints and variables in the equivalent model are much fewer than that in the initially provided one. Hence, the proposed shortcut model can be easily utilized according to Iskander's approach, especially for large scale problems.
\end{abstract}

JEL classification numbers: C61, C63.

Keywords: Alternative fuzzy goals, Zero-one fuzzy goal programming, Mixed zero-one fuzzy goal programming.

\section{Introduction}

Iskander [1] provided an approach in fuzzy goal programming where the original fuzzy goals can be replaced by their corresponding alterative ones based on the satisfaction of certain situation conditions. Therefore, with reference to his stated fuzzy goal programming problem as well as all the definitions, the crisp non-linear mixed zero-one goal program has been presented as follows:

\footnotetext{
${ }^{1}$ Faculty of Business Administration, Economics and Political Science, The British University in Egypt, 11837, Egypt.
}

Article Info: Received: January 2, 2022. Revised: January 23, 2022.

Published online: January 31, 2022. 
Lexicographically minimize $\left\{\sum_{i \in I_{p}} d_{i}+\sum_{\substack{i \in I_{p} \\ i \in G}} d_{i}^{0}: p=1,2, \ldots, P\right\}$

subject to:

$$
\begin{aligned}
& L_{i} f_{i}(X, Y)-L_{i} u_{i}+d_{i} \geq 1, i=1,2, \ldots, m_{1} ; i \notin G, \\
& K_{i} v_{i}-K_{i} f_{i}(X, Y)+d_{i} \geq 1, i=m_{1}+1, m_{1}+2, \ldots, m ; i \notin G \\
& r_{j}=R_{j}(X), j=1,2, \ldots, J, \\
& L_{i} f_{i}(X, Y) r_{j}-L_{i} u_{i} r_{j}+d_{i} \geq r_{j}, i \in G_{j} ; i=1,2, \ldots, m_{1} ; j=1,2, \ldots, J, \\
& K_{i} v_{i} r_{j}-K_{i} f_{i}(X, Y) r_{j}+d_{i} \geq r_{j}, i \in G_{j} ; i=m_{1}+1, m_{1}+2, \ldots, m ; j=1,2, \ldots, J, \\
& L_{i}^{\mathrm{o}} f_{i}^{\mathrm{o}}(X, Y)\left(1-r_{j}\right)-L_{i}^{\mathrm{o}} u_{i}^{\mathrm{o}}\left(1-r_{j}\right)+d_{i}^{\mathrm{o}} \geq\left(1-r_{j}\right), i \in G_{j} ; i=1,2, \ldots, m_{1} ; j=1,2, \ldots, J,(7) \\
& K_{i}^{\mathrm{o}} v_{i}^{\mathrm{o}}\left(1-r_{j}\right)-K_{i}^{\mathrm{o}} f_{i}^{\mathrm{o}}(X, Y)\left(1-r_{j}\right)+d_{i}^{\mathrm{o}} \geq\left(1-r_{j}\right), i \in G_{j} ; i=m_{1}+1, m_{1}+2, \ldots, m ; \\
& j=1,2, \ldots, J
\end{aligned}
$$

$\Phi_{s}(X, Y) \leq 0, s=1,2, \ldots, S$,

$0 \leq d_{i} \leq 1, i=1,2, \ldots, m$,

$0 \leq d_{i}^{\mathrm{o}} \leq 1, i \in G$

$X, r_{j} \in\{0,1\}, j=1,2, \ldots, J$

$Y \geq 0$.

\section{The Equivalent Shortcut Model}

Iskander's model (1)-(13) has been linearized by two approaches. The first is used to linearize the product of binary variables. The second (Chang's approach) is utilized when a non-negative variable is multiplied by a binary variable [2].

The proposed equivalent shortcut model requires that in the above model (1)-(13), constraints (5)-(8) should be respectively replaced by the following constraints:

$$
\begin{aligned}
& \theta\left(1-r_{j}\right)+L_{i} f_{i}(X, Y)-L_{i} u_{i}+d_{i} \geq 1, i \in G_{j} ; i=1,2, \ldots, m_{1} ; j=1,2, \ldots, J, \\
& \theta\left(1-r_{j}\right)+K_{i} v_{i}-K_{i} f_{i}(X, Y)+d_{i} \geq 1, i \in G_{j} ; i=m_{1}+1, m_{1}+2, \ldots, m ; j=1,2, \ldots, J, \\
& \theta r_{j}+L_{i}^{\mathrm{o}} f_{i}^{\mathrm{o}}(X, Y)-L_{i}^{\mathrm{o}} u_{i}^{\mathrm{o}}+d_{i}^{\mathrm{o}} \geq 1, i \in G_{j} ; i=1,2, \ldots, m_{1} ; j=1,2, \ldots, J,
\end{aligned}
$$


$\theta r_{j}+K_{i}^{\mathrm{o}} v_{i}^{\mathrm{o}}-K_{i}^{\mathrm{o}} f_{i}^{\mathrm{o}}(X, Y)+d_{i}^{\mathrm{o}} \geq 1, i \in G_{j} ; i=m_{1}+1, m_{1}+2, \ldots, m ; j=1,2, \ldots, J$,

where $\theta$ is a large positive number. It is obvious that the non-linear constraints (5)-(8) are respectively replaced by the linear constraints (14)-(17). Hence, the Chang's linearization approach is not required to linearize the shortcut model, just the first approach is utilized to linearize constraint set (4).

\section{Illustrative Implementation}

In this section, the same numerical example, considered by Iskander [1], is used to illustrate the implementation of the shortcut model. This example assumed the following mixed zero-one fuzzy goal program:

(g) $50 x_{1}+30 x_{2}+40 x_{3}+4 y_{1}+6 y_{2}>60$

$\left(g_{2}\right) 20 x_{1}+40 x_{2}+10 x_{3}<40$

$\left(g_{3}\right) 10 y_{1}+6 y_{2}<30$

$$
\begin{aligned}
& x_{1}+x_{2}+x_{3} \geq 1, \\
& 3 y_{1}+2 y_{2} \geq 11, \\
& x_{1}, x_{2}, x_{3} \in\{0,1\}, \\
& y_{1}, y_{2} \geq 0 .
\end{aligned}
$$

The alternative fuzzy goals for the original three ones $\left(g_{1}, g_{2}, g_{3}\right)$ are respectively as follows:

$\left(g_{1}^{\circ}\right) 40 x_{1}+35 x_{2}+40 x_{3}+5 y_{1}+5 y_{2}>65$

$\left(g_{2}{ }^{\circ}\right) 20 x_{1}+30 x_{2}+20 x_{3} \underset{\sim}{<50}$

$\left(g_{3}{ }^{\mathrm{o}}\right) 7 y_{1}+8 y_{2}<25$.

The tolerance limits for the original fuzzy goals and the alternative ones are respectively $(50,45,35)$ and $(60,55,35)$. Two priority levels are used. The first is assigned to the first goal, while the second is assigned to the second and third goals. Finally, one situation condition is stated, whereas the original three fuzzy goals are considered if both $x_{1}$ and $x_{3}$ 
are equal to one, otherwise the alternative ones are considered. Then, the linearized crisp shortcut mixed zero-one goal program is stated as follows:

Lexicographically minimize $\left\{d_{1}+d_{1}{ }^{\mathrm{o}}, d_{2}+d_{2}{ }^{\mathrm{o}}+d_{3}+d_{3}{ }^{\mathrm{o}}\right\}$

subject to:

$\theta(1-r)+5 x_{1}+3 x_{2}+4 x_{3}+0.4 y_{1}+0.6 y_{2}-6+d_{1} \geq 0$

$\theta(1-r)+8-4 x_{1}-8 x_{2}-2 x_{3}+d_{2} \geq 0$

$\theta(1-r)+6-2 y_{1}-1.2 y_{2}+d_{3} \geq 0$,

$\theta r+8 x_{1}+7 x_{2}+8 x_{3}+y_{1}+y_{2}-13+d_{1}{ }^{\mathrm{o}} \geq 0$,

$\theta r+10-4 x_{1}-6 x_{2}-4 x_{3}+d_{2}{ }^{\circ} \geq 0$,

$\theta r+2.5-0.7 y_{1}-0.8 y_{2}+d_{3}{ }^{\circ} \geq 0$

$x_{1}+x_{3}-1 \leq r \leq\left(x_{1}+x_{3}\right) / 2$,

$x_{1}+x_{2}+x_{3} \geq 1$

$3 y_{1}+2 y_{2} \geq 11$

$0 \leq d_{1}, d_{2}, d_{3}, d_{1}{ }^{\mathrm{o}}, d_{2}{ }^{\mathrm{o}}, d_{3}{ }^{\mathrm{o}} \leq 1$,

$r, x_{1}, x_{2}, x_{3} \in\{0,1\}$,

$y_{1}, y_{2} \geq 0$.

However, there is only one situation condition, the number of constraints and variables in this shortcut numerical model is less than that in its equivalent original one which has been presented by Iskander [1].

\section{References}

[1] Iskander, M.G. (2015). A suggested approach in mixed zero-one fuzzy goal programming. Journal of Computations \& Modelling, 5(2), pp. 49-58.

[2] Chang, C.T. (2007). Binary fuzzy goal programming. European Journal of Operational Research, 180(1), pp. 29-37. 